\title{
Case Report Severe case of Mediterranean spotted fever in
Greece with predominantly neurological features
}

\author{
Correspondence \\ Konstantina Tzavella \\ ktzavell@med.auth.gr
}

Received 19 September 2005 Accepted 29 October 2005

\author{
Konstantina Tzavella, ${ }_{1}^{1}$ loannis S. Hatzizisis, ${ }^{1}$ Aikaterini Vakali, ${ }^{2}$ \\ Kalliopi Mandraveli, ${ }^{3}$ Dimitrios Zioutas ${ }^{4}$ and Stella Alexiou-Daniel ${ }^{3}$ \\ First Propedeutic Medical Department', Department of Radiology², First Department of \\ Microbiology ${ }^{3}$ and Department of Cardiology 4 , AHEPA University Hospital, Aristotle University \\ of Thessaloniki, Thessaloniki, Greece
}

\begin{abstract}
Rickettsia conorii is endemic in Greece, though only a few cases of infection have been published to date. The case of a 58-year-old man from northern Greece with a severe form of Mediterranean spotted fever and rapid neurological deterioration is presented here. The patient received antibiotic treatment with doxycycline, showing immediate clinical and laboratory improvement. Diagnosis was confirmed later, during the second week after disease onset, by detection of elevated titres of $\operatorname{lgM}$ and $\lg G$ antibodies against $R$. conorii using an indirect immunofluorescence assay.
\end{abstract}

\section{Case report}

A 58-year-old retired and previously healthy man presented himself at a peripheral hospital in northern Greece with a sudden onset of chest pain at rest and headache. Clinical, laboratory and imaging investigation, including electrocardiogram, troponin T test, stress test and echocardiogram, showed no pathological findings; the chest pain receded spontaneously and the patient was released after 2 days. One day later, at home, the patient developed high fever $\left(39^{\circ} \mathrm{C}\right)$ with persisting headache and diffuse myalgia. He received amoxycillin-clavulanic acid orally, and showed only a slight improvement of his symptoms. Additionally, a facial and upper limb maculopapular rash occurred 2 days later, which was considered to be an allergic side effect of the antibiotic therapy. The antibiotics were discontinued and the patient received antihistamines. He still complained of headache, myalgia and subfebrile temperatures. The next day ( 6 days after disease onset) the patient suddenly developed confusion, for which reason he was transferred to our department immediately. Clinical examination at admission revealed an axillary body temperature of $37.8{ }^{\circ} \mathrm{C}$ and a fading diffuse macupapular rash with no signs of lymphadenopathy. Neurological examination of the patient showed confusion, ataxia and dysarthria without neck stiffness or any focal neurological findings. Although a history of tick exposure was denied, a black necrotic scabbed lesion of $5 \mathrm{~mm}$ in diameter was found on the patient's left thumb.

Initial laboratory tests showed elevated levels of C-reactive protein, up to $8 \cdot 5 \mathrm{mg} \mathrm{dl}^{-1}$ (normal range $<0 \cdot 8 \mathrm{mg} \mathrm{dl}^{-1}$ ), and procalcitonin, up to $1.6 \mathrm{ng} \mathrm{dl}^{-1}$ (normal range $\left.<0 \cdot 5 \mathrm{ng} \mathrm{dl}^{-1}\right)$, accelerated erythrocyte sedimentation rate

Abbreviation: MSF, Mediterranean spotted fever. $\left(70 \mathrm{~mm} \mathrm{~h}^{-1}\right)$, thrombocytopenia (70000 platelets $\left.\mu \mathrm{l}^{-1}\right)$ with normal white blood cell count and haemoglobin, impaired renal function $\left(1.9 \mathrm{mg}\right.$ creatinine $\mathrm{dl}^{-1}$, normal range $0 \cdot 5-1 \cdot 4 \mathrm{mg} \mathrm{dl}^{-1} ; 101 \mathrm{mg}$ serum urea $\mathrm{dl}^{-1}$, normal range $10-50 \mathrm{mg} \mathrm{dl}^{-1}$ ), elevated lactate dehydrogenase (1016 $\mathrm{U} \mathrm{l}^{-1}$, normal range 240-480 $\mathrm{U} \mathrm{l}^{-1}$ ), $\gamma$-glutamyl transpeptidase (183 $\mathrm{U} \mathrm{l}^{-1}$, normal range 8-65 $\mathrm{U} \mathrm{l}^{-1}$ ), alanine aminotransferase $\left(172 \mathrm{U} \mathrm{l}^{-1}\right.$, normal range $<38 \mathrm{U}$ $\mathrm{l}^{-1}$ ), aspartate aminotransferase (205 $\mathrm{U} \mathrm{l}^{-1}$, normal range $<41 \mathrm{U} \mathrm{l}^{-1}$ ) and alkaline phosphatase (509 $\mathrm{U} \mathrm{l}^{-1}$, normal range 40-129 $\left.\mathrm{U} \mathrm{l}^{-1}\right)$. Blood-gas analysis revealed hypoxaemia $\left(\mathrm{pO}_{2} 60 \mathrm{~mm} \mathrm{Hg}, \mathrm{pCO}_{2} 35 \mathrm{~mm} \mathrm{Hg}, \mathrm{pH} 7 \cdot 4\right)$. The cerebrospinal fluid showed a slight pleocytosis (12 cells $\mathrm{mm}^{-3}, 90 \%$ polymorphonuclear) and an increased level of protein $\left(53 \mathrm{mg} \mathrm{dl}^{-1}\right)$. A CT (computed tomography) scan and ultrasound of the abdomen showed mild enlargement of liver and spleen without lymphadenopathy. Chest X-ray, CT scan and magnetic resonance imaging of the head, as well as an encephalogram, were normal.

The patient remained haemodynamically stable, but deteriorated neurologically within the first $24 \mathrm{~h}$ after admission, in spite of additional intravenous therapy with dexamethasone and mannitol. Due to clinical findings (diffuse macupapular rash, fever, headache, myalgia, inoculation eschar) and laboratory findings (thrombocytopenia, elevated alanine and aspartate aminotransferase) a rickettsial infection was suggested, so antibiotic therapy with doxycycline (200 mg per day, orally) was started on the eighth day after disease onset. Only $24 \mathrm{~h}$ later a remarkable improvement was seen in the patient's neurological symptoms and the pathological laboratory findings. The rash temporarily worsened and became petechial, but disappeared approximately 7 days later. 
At 12 days after disease onset an indirect immunofluorescent antibody test revealed elevated IgM and IgG titres to Rickettsia conorii ( $1: 512$ and $1: 64$, respectively) with rising titres, up to $1: 2048$ and $1: 1024$, respectively, 2 weeks later. No other source of infection was found. There was no growth on routine microbiological culture of blood, urine and cerebrospinal fluid; typhoid fever, endemic typhus and toxoplasmosis were ruled out.

The patient was discharged 14 days after admission, receiving doxycycline for a total of 15 days. He also remained asymptomatic without any pathological laboratory findings at control examinations 6 and 9 weeks later. There was a further increase of $\operatorname{IgG}$ titres against $R$. conorii $(>1: 2056)$, while the IgM titre declined continuously (final titre $<1: 64)$.

\section{Discussion}

Mediterranean spotted fever (MSF), also known as 'boutonneuse' fever, is prevalent in Mediterranean countries, Africa, India, south-western and south central Asia, east Russia, and in areas around the Black Sea. It is caused by $R$. conorii, an obligate intracellular Gram-negative bacterium, and transmitted by the brown dog tick Rhipicephalus sanguineus in urban and suburban areas (Brouqui et al., 2004; Martinez \& Cossart, 2004; Raoult \& Roux, 1997). Typical clinical features include fever, myalgia, headache, generalized maculopapular rash and an inoculation eschar ('tache noir') at the site of the tick bite (Raoult \& Roux, 1997). MSF typically follows a benign course, but in about $6 \%$ of the cases it may cause severe illness involving multiple organs, with a mortality of $2 \cdot 5-5 \%$ (Raoult et al., 1986; Yagupsky \& Wolach, 1993). Neurological involvement has been reported in $28 \%$ of patients and constitutes a negative prognostic factor for the outcome of the disease (Alioua et al., 2003).

MSF is significantly seasonal, with most cases appearing during spring and summer months (Raoult \& Roux, 1997; Alexiou-Daniel et al., 2003). Seroepidemical investigations showed a high seroprevalence of $R$. conorii among the healthy Greek population (Daniel et al., 2002); however, only few cases of MSF have recently been reported (AlexiouDaniel et al., 2003; Spengos et al., 2005).

In this report we presented a severe case of MSF with predominantly neurological features. The infection developed in July and the patient was living in an urban area of northern Greece without contact with domestic dogs. The severity of the illness was probably due to the delayed initiation of the appropriate antibiotic therapy with doxycycline. Our patient developed the symptoms gradually during the first week of the disease, so that he was treated for each one (e.g. chest pain, a possible initial symptom of myalgia, fever, rash) separately, while the underlying disease remained unrecognized. The initial flu-like symptoms of MSF may confuse physicians, and the common practice of prescribing $\beta$-lactams as empirical therapy may contribute to a delay in the diagnosis of boutonneuse fever and result in life-threatening complications or permanent disabilities in some infected individuals (Jensenius et al., 2004). Early recognition of the disease and immediate administration of the appropriate antibiotic therapy is essential for rapid recovery and prevention of complications, but confirmation of diagnosis during the acute phase is difficult. For this reason, diagnosis of the disease should initially be based on epidemiologic criteria as well as on clinical findings and laboratory findings (e.g. thrombocytopenia, elevated alanine and aspartate aminotransferase), according to the diagnostic criteria described by Brouqui et al. (2004).

The diagnosis of boutonneuse fever is usually confirmed by serological testing using an immunofluorescence assay, the reference technique in most laboratories, to detect IgM and IgG antibodies against $R$. conorii in acute and convalescent sera (La Scola \& Raoult, 1997; Brouqui et al., 2004). Detection of both antibodies can usually be made 7-15 days after disease onset, and therefore provides a retrospective diagnosis. In our case IgM and $\operatorname{IgG}$ antibodies against $R$. conorii were detected during the second week of the illness, showing a titre increase in excess of fourfold 2 weeks later.

Despite the delayed beginning, our patient recovered promptly after administration of doxycycline, currently the standard therapeutic regimen for MSF (Jensenius et al., 2004).

In conclusion, due to the high seroprevalence of $R$. conorii in Greece we believe that MSF has been underrecognized and underreported so far, and may occur as a severe illness in our country. Not only domestic, but also foreign, physicians should be aware and remember the possibility of the disease for all febrile patients living in or recently travelling from a tick-infected area in Greece, even in the absence of an inoculation eschar and skin rash.

\section{References}

Alexiou-Daniel, S., Tea, A., Ilonidis, G. \& Antoniadis, A. (2003). A case of pleurisy associated with antibodies to Rickettsia conorii. Clin Microbiol Infect 9, 437-440.

Alioua, Z., Bourazza, A., Lamsyah, H., Erragragui, Y., Boudi, O., Karouach, K., Ghfir, M., Mossedaq, R. \& Sedrati, O. (2003). Neurological feature of Mediterranean spotted fever: a study of four cases. Rev Med Interne 24, 824-829.

Brouqui, P., Bacellar, F., Baranton, G. \& 22 other authors (2004). ESCMID study group report. Guidelines for the diagnosis of tick-borne bacterial diseases in Europe. Clin Microbiol Infect 10, 1108-1132.

Daniel, S. A., Manika, K., Arvanitidou, M. \& Antoniadis, A. (2002). Prevalence of Rickettsia conorii and Rickettsia typhi infections in the population of northern Greece. Am J Trop Med Hyg 66, 76-79.

Jensenius, M., Fournier, P. E. \& Raoult, D. (2004). Rickettsioses and the international traveller. Clin Infect Dis 39, 1493-1499.

La Scola, B. \& Raoult, D. (1997). Laboratory diagnosis of rickettsioses: current approaches to diagnosis of old and new rickettsial diseases. J Clin Microbiol 35, 2715-2727.

Martinez, J. J. \& Cossart, P. (2004). Early signaling events involved in the entry of Rickettsia conorii into mammalian cells. J Cell Sci 117, 5097-5106. 
Raoult, D. \& Roux, V. (1997). Rickettsioses as paradigms of new or emerging infectious diseases. Clin Microbiol Rev 10, 694-719.

Raoult, D., Weiller, P. J., Chagnon, A., Chaudet, H., Gallais, H. \&

Casanova, P. (1986). Mediterranean spotted fever: clinical, laboratory and epidemiological features of 199 cases. Am J Trop Med Hyg $35,845-850$.
Spengos, K., Stouraitis, G., Voumvourakis, K., Zambelis, T. \& Karandreas, N. (2005). Motor and sensory polyneuritis with distal conduction failure as uncommon complication of an acute Rickettsia conorii infection. J Neurol Sci 234, 113-116.

Yagupsky, P. \& Wolach, B. (1993). Fatal Israeli spotted fever in children. Clin Infect Dis 17, 850-853. 